\title{
Controlled Drug Administration by a Fractional PID *
}

\author{
Pantelis Sopasakis *,** and Haralambos Sarimveis ** \\ * IMT Institute for Advanced Studies Lucca, Piazza San Ponziano 6, \\ Lucca 55100, Italy (Tel: +390583 4326 710; e-mail: \\ pantelis.sopasakis@imtlucca.it). \\ ** School of Chemical Engineering, National Technical University of \\ Athens, 9 Heroon Polytechneiou Street, 15780 Zografou Campus, \\ Athens, Greece (Tel: +30 210 7723237, e-mail: \\ hsarimv@central.ntua.gr)
}

\begin{abstract}
Amiodarone is an antiarrhythmic drug that exhibits highly complex and nonexponential dynamics whose controlled administration has important implications for its clinical use especially for long-term therapies. Its pharmacokinetics has been accurately modelled using a fractional-order compartmental model. In this paper we design a fractional-order PID controller and we evaluate its dynamical characteristics in terms of the stability margins of the closed loop and the ability of the controlled system to attenuate various sources of noise and uncertainty.
\end{abstract}

Keywords: Biomedical control; Biomedical systems; Pharmacokinetics and drug delivery; Control of physiological and clinical variables; Physiological model; Fractional control.

\section{INTRODUCTION}

\subsection{Motivation}

It is characteristic how Machado et al. [2011] open their review on the recent history of fractional calculus saying that "fractional calculus may be considered an old and yet novel topic." Although lots of mathematicians have worked on this field, many fundamental questions remain unanswered. From the point of view of applied research the field is nowadays very active - it has been only the last few decades that the scientific community turned to fractional calculus seeking for a modeling solution for phenomena that ordinary calculus had a hard time to approach. Such complex phenomena are biological membranes, viscoelastic materials, certain electrochemical processes and biomedical phenomena (see Magin [2006]).

In 2010, Kytariolos et al. [2010] introduced fractional dynamics in the field of pharmacokinetic modelling pointing out the main reasons for the failure of the classical IVIVC (In-Vitro In-Vivo Correlations) theory. The first step to this direction was taken by Dokoumetzidis and Macheras [2009]. In practice, non-linearities, anomalous diffusion, fractional-order kinetics, diffusion accross fractal manifolds, synergetic and competitive action and a great many other factors render this approach not applicable (see Dokoumetzidis and Macheras [2008]). Recently, a significant number of relevant publications has emerged; see Popović et al. [2012], Verotta [2010a,b], Pereira [2010], Dokoumetzidis et al. [2010b], Popović et al. [2010].

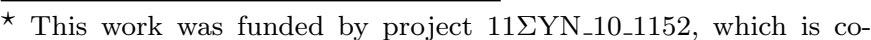
financed by the European Union and Greece, Operational Program 'Competitiveness \& Entrepreneurship', NSFR 20072013 in the context of GSRT-National action "Cooperation".
}

Fractional-order derivatives - which we shall define properly in the sequel - although seem to be a mere mathematical construction and a method of nonlinear modelling, possess, in fact, physical meaning and occur naturally through physical phenomena. Hilfer [2000a] called attention on the fact that such operators emerge naturally from the study of anomalous diffusion and other phenomena (Hilfer [2000b]).

Fractional dynamics can be cast as Physiologically Based Pharmacokinetic Models (PBPK) with fractional-order derivatives (see Dokoumetzidis et al. [2010a]) where the mass balance equations are rewritten using fractionalorder derivatives. This offers a mechanistic understanding of the interplay among the main factors of drug distribution, allows us to draw individualized concentrationtime profiles and study drug-drug interactions using the fractional calculus approach. The "fractionalisation" of pharmacokinetic equations though has to be done in a way so that the mass preservation holds.

The problem of drug administration is purely a control problem where the aim is to keep the drug concentration at certain organs in the body close to the desired therapeutical set-points while the concentration in other organs and tissues does not exceed certain safety limits (see Sarimveis et al. [2009]). The manipulated variable is the administered dosage and the controlled variable is the concentration of the drug in some tissue or organ of the body.

It is nowadays clear that accurate pharmacokinetic modelling is essential for the understanding of the action of a drug and is of major importance for of the establishment of an efficient therapeutical administration course. Feedback control is concededly a practice that enjoys wide acceptance in medicine (see Sopasakis and Sarimveis [2012] 
and Dua et al. [2006]), though using linear integer-order pharmacokinetic models.

In this paper we employ a fractional pharmacokinetic model to describe the distribution of Amiodarone upon intravenous administration, which has proven to be very accurate when juxtaposed to actual data. Amiodarone follows anomalous, non-exponential pharmacokinetic dynamics with major clinical implications as the drug may overaccumulate in a long-term administration course. We propose the use of a fractional PID controller and we explore the dynamical characteristics of the closed-loop system.

\subsection{Fractional Dynamical Systems}

The most exotic property of non-integer order derivatives is that they are not local properties. The calculation of $\left(D^{n} f\right)\left(x_{0}\right)$ (with $n \in \mathbb{N}$ ) requires the knowledge of $f$ in an arbitrarily small region around $x_{0}$, say $\left(x_{0}-\right.$ $\left.\varepsilon, x_{0}+\varepsilon\right)$; this is not true for non-integer order derivatives $\left(D^{a} f\right)\left(x_{0}\right)$ (with $\left.a \in \mathbb{R}\right)$. This property proves invaluable when modeling phenomena with memory or other complex dynamics. Using fractional calculus, one obtains some peripheral vision in contrast to the local approach that ordinary derivatives offer and the process is now modeled using integro-differential equations.

Let us first define the generalised Riemann-Liouville fractional-order integral operator which extends the $n$-th order $(n \in \mathbb{N})$ integral $I^{n}$ which is given by the Cauchy formula:

$$
\left(I^{n} f\right)(t)=\frac{1}{(n-1) !} \int_{0}^{t}(t-\tau)^{n-1} f(\tau) \mathrm{d} \tau, t \geq 0 .
$$

Using the property of the Gamma function that $\Gamma(n)=$ $(n-1)$ ! for all $n \in \mathbb{N}$ we arrive at the following generalised integral of real order $\alpha \in \mathbb{R}$ :

$$
\left(I^{\alpha} f\right)(t)=\frac{1}{\Gamma(\alpha)} \int_{0}^{t}(t-\tau)^{\alpha-1} f(\tau) \mathrm{d} \tau, t \geq 0 .
$$

For $\alpha \in \mathbb{R}$ let us denote by $m=\lceil\alpha\rceil$ the smallest natural number $m$ so that $m \geq \alpha$. The following operator is known as the Caputo derivative of order $\alpha$ :

$$
\left(D^{\alpha} f\right)(t)=I^{m-\alpha} \frac{\mathrm{d}^{m} f(t)}{\mathrm{d} t^{m}} .
$$

This operator enjoys a number of good properties such that it extends the integer-order derivative to arbitrary real orders, it preserves analiticity, it is a linear operator, it has the semigroup property (i.e., $D^{\alpha} D^{\beta} f=D^{\alpha+\beta} f$ ) and $D^{0}$ is the identity operator. It is of central importance that the Laplace transformation of the Caputo fractional derivative can be calculated by the following formula:

$$
\mathcal{L}\left[D^{\alpha} f\right](s)=s^{\alpha} F(s)-\left.\sum_{k=0}^{m-1} s^{\alpha-k-1} \frac{\mathrm{d}^{k} f}{\mathrm{~d} t^{k}}\right|_{0},
$$

where $F(s)=(\mathcal{L} f)(s)$.

Such generalized derivation operators give rise to the corresponding class of functional relations known as fractional differential equations and have the general form:

$$
F\left(t, x, D^{\alpha_{1}} x, \ldots, D^{\alpha_{n}} x\right)=0 .
$$

Dynamical systems with fractional derivatives can be represented in the following form:

$$
H\left(D^{\alpha_{1}}, \ldots, D^{\alpha_{n}}\right) x=T\left(D^{\beta_{1}}, \ldots, D^{\beta_{m}}\right) u
$$

where $x$ and $u$ stand for the output and input variables. In this paper we consider the case of linear fractional dynamical systems, i.e., we assume that $H$ and $T$ are linear functions. Linear fractional dynamical systems can be represented in the Laplace domain in terms of their tranfer functions:

$$
G(s)=\frac{X(s)}{U(s)}=\frac{P(s)}{Q(s)},
$$

where $P$ and $Q$ are fractional polynomials, i.e., functions of the form $P(s)=\sum_{i=0}^{n} a_{i} s^{b_{i}}$ with $b_{i}>0$ for all $i=$ $0, \ldots, n$, and $X(s)=(\mathcal{L} x)(s)$ and $U(s)=(\mathcal{L} u)(s)$. A very interesting fact is that the achievement of equilibrium for the output of the system (i.e., $\dot{x}(t)=0$ ) does not imply that the input is also in equilibrium (i.e., $\dot{u}(t)=0$ ). This, however, does not hinder us from studying the stability of such systems in the BIBO (Bounded-Input BoundedOutput) sense. To this end, let us define the open-loop transfer function of the system:

$$
G_{\mathrm{ol}}(s)=G(s) G_{\mathrm{c}}(s),
$$

where $G_{c}(s)$ is the transfer function of the controller. The closed-loop transfer function, which relates the set-point $x^{s p}$ with the response $x$, is:

$$
G_{\mathrm{cl}}(s)=\frac{G_{\mathrm{c}}(s) G(s)}{1+G_{\mathrm{c}}(s) G(s)} .
$$

Various stability criteria have been postulated; in this paper we shall use a Bode-type criterion which applies to $G_{\mathrm{ol}}$ (see Monje et al. [2010]):

Criterion 1. (BIBO Stability Criterion). Assume there exists a frequency $\omega_{\text {co }}>0$ so that $\arg G_{\mathrm{ol}}\left(\imath \omega_{\mathrm{co}}\right)=-\pi$ (This will be referred to as the crossover frequency of the system). If $\left|G_{\mathrm{ol}}\left(\imath \omega_{\mathrm{co}}\right)\right|<0 \mathrm{db}$, then $G_{\mathrm{cl}}$ is BIBO-stable.

\subsection{Numerical Simulations}

In certain simple cases, the solution of fractional differential equations and initial value problems can be performed analytically - usually in terms of the Mittag-Leffler special function:

$$
\mathcal{E}_{a, b}(z)=\sum_{k \in \mathbb{N}} \frac{z^{k}}{\Gamma(a k+b)} .
$$

For instance, it is:

$$
\mathcal{L}^{-1} \frac{s^{a-b}}{s^{a}-\lambda}=t^{b-1} \mathcal{E}_{a, b}\left(\lambda t^{a}\right)
$$

In a more general case, however, it is not possible to derive analytical expressions for the solution. Aoun et al. [2004] provide a thorough review of numerical methods for the solution of fractional differential equations. A very widespread approach consists in approximating the fractional dynamics by some integer one. The Oustaloup filter (see Oustaloup et al. [2000], Merrikh-Bayat [2012]) is a well established technique for the approximation of fractional transfer functions by integer ones in a specified range of operating frequencies.

\subsection{Fractional-PID Controllers}

Fractional PID controllers were introduced by Podlubny [1999] and are generalisations of the standard PID. A 
fractional PID controller, or $\mathrm{PI}^{\lambda} \mathrm{D}^{\mu}$ has a tranfer function of the following form:

$$
G_{\mathrm{c}}(s)=K_{p}+\frac{K_{i}}{s^{\lambda}}+K_{d} s^{\mu},
$$

where $K_{p}, K_{i}, K_{d}, \lambda$ and $\mu$ are positive tuning parameters, and it produces the control action:

$$
u(t)=K_{p} \epsilon(t)+K_{i} I^{\lambda} \epsilon(t)+K_{d} D^{\mu} \epsilon(t),
$$

where $\epsilon(t)$ is the deviation of the system's output from the set-point $x_{\mathrm{sp}}(t)$ defined as $\epsilon(t):=x_{\mathrm{sp}}(t)-x(t)$. A fractional PID controller involves 5 tuning parameters so it offers greater flexibility by enabling us to determine both its low and high-frequency gains. One of the most well-established methods for the tuning of fractional PID controller is the minimisation of a performance index involving the step response of the closed-loop sytem. A standard choice is the ITAE (Integral Time Absolute Error) index defined as:

$$
J_{\text {itae }}:=\int_{0}^{\infty} \tau \epsilon(\tau) \mathrm{d} \tau
$$

This leads to the formulation of the following optimisation problem:

$$
J_{\text {itae }}^{\star}:=\min _{K_{p}, K_{i}, K_{d}, \lambda, \mu} J_{\text {itae }},
$$

provided that the choice of parameters leads to a stable closed-loop system. Such optimisation problems are nonconvex and their numerical solution depends on the initial estimate; it is therefore recommended to try a multiplitude of initial values. Monje et al. [2010] suggest that there may be more than one acceptable parametrization.

The optimisation of the ITAE criterion can be carried out along with some additional requirements so as to guarantee certain desirable dynamical characteristics for the closedloop. Valério and da Costa [2006] prescribe a set of 5 requirements for the tuning of fractional PID controllers which attenuate the in-loop noise and damp external noises. Noise rejection in the closed loop is guaranteed by the following condition:

$$
M_{h}:=\left|G_{\mathrm{cl}}\left(\imath \omega_{h}\right)\right|<\eta,
$$

where $\omega_{h}$ is an arbitrarily chosen high frequency and $\eta$ is a design parameter. We also require that there should be a crossover frequency $\omega_{\text {co }}$ and the gain margin be equal to a given value (greater than 1). What is the same we postulate a similar requirement for the phase margin. The controller has also to compensate parametric uncertainties that can be the cause of inaccurate modelling or timevarying dynamics of the controlled process. To enforce this robustness qualification we require that:

$$
M_{z}:=\left.\frac{\mathrm{d}}{\mathrm{d} \omega} \arg \left(G_{\mathrm{ol}}(\imath \omega)\right)\right|_{\omega=\omega_{\mathrm{co}}}<\zeta,
$$

where $\zeta>0$ is a design parameter. Finally, the closed-loop system has to compensate for output disturbances, which are in principle of low frequency (see Åström [2002]). To this end, we require that the sensitivity function of the system, defined as

$$
G_{\mathrm{sens}}(s):=\frac{1}{1+G_{\mathrm{ol}}(s)},
$$

exhibit low gain at low frequencies, i.e.,

$$
M_{\ell}:=\left|G_{\text {sens }}\left(\imath \omega_{\ell}\right)\right|<\vartheta
$$

where $\omega_{\ell}$ is some, arbitrarily chosen, low frequency and $\vartheta$ is a properly small design parameter.
Table 1. PK Parameters of Amiodarone

\begin{tabular}{lll}
\hline Parameter & Value & \\
\hline$a$ & 0.5870 & \\
$k_{10}$ & 1.4913 day & 1 \\
$k_{12}$ & 2.9522 day & 1 \\
$k_{21}$ & 0.4854day & \\
\hline
\end{tabular}

These requirements need to be taken into account while tuning a fractional PID controller. This way, the optimisation problem (15) is solved subject to the nonlinear constraints (16) to (19).

\section{FEEDBACK CONTROL OF AMIODARONE ADMINISTRATION}

Amiodarone is an antiarrhythmic agent (see Kühlkamp et al. [1999]) which can be administered either intravenously (i.v.) or orally. Amiodarone is well-known for its highly nonlinear non-exponential dynamics and singular long-term accumulation pattern. Recently, Dokoumetzidis et al. [2010a] modelled the pharmacokinetic distribution of Amiodarone with a fractional compartmental model following a single i.v. and a single oral dose. The compartmental topology of the model is presented in Figure 1.

Let $A_{1}$ and $A_{2}$ be the amounts of Amiodarone (in ng) in the plasma and the tissues respectively and $u$ be the administration rate (in ng/day). We assume that the drug is administered directly into the central (plasma) compartment while the control objective is the concentration of the drug in the tissues attains a prescribed value (setpoint). The fractional dynamical model we employ reads as follows:

$$
\begin{aligned}
& \frac{\mathrm{d} A_{1}}{\mathrm{~d} t}=-\left(k_{12}+k_{10}\right) A_{1}+k_{21} \cdot D^{1-a} A_{2}+u, \\
& \frac{\mathrm{d} A_{2}}{\mathrm{~d} t}=k_{12} A_{1}-k_{21} \cdot D^{1-a} A_{2},
\end{aligned}
$$

with $a<1$. The terms " $k_{21} \cdot D^{1-a} A_{2}$ " correspond to the fractional-order diffusion of Amiodarone from the tissues to the central compartment, the term " $k_{12} A_{1}$ " is the rate at which Amiodarone is transfered from the central compartment to the tissues and " $k_{10} A_{1}$ " defines the excretion rate. This dynamics is graphically presented in Fig. 1. The parameters of the aforementioned model are presented in Table 1 . The transfer function of the system which links the administration rate $U(s)=\mathcal{L} u(t)$ to the concentration of Amiodarone in the tissues $\hat{A}_{2}(s)=$ $\mathcal{L} A_{2}(t)$ is:

$$
G(s)=\frac{\frac{1}{k_{10}}\left(\frac{1}{k_{21}} s^{a}+1\right)}{\frac{1}{k_{10} k_{21}} s^{a+1}+\frac{1}{k_{10}} s+\frac{k_{10}+k_{12}}{k_{10} k_{21}} s^{a}+1}
$$

Before the commencement of the administration course we assume that the initial concentration of Amiodarone in the organism is zero, i.e., $A_{1}(t)=A_{2}(t)=0$ for all $t \leq 0$.

We tuned a fractional PID controller for the control of the concentration of Amiodarone in the central compartment the parameters of which were chosen using the ITAE criterion along with the additional constraints outlined above. We chose the characteristic frequencies $\omega_{\ell}=0.01 \mathrm{rad} /$ day, and $\omega_{h}=100 \mathrm{rad} /$ day and required that $\vartheta=-10 \mathrm{db}, \eta=$ $-20 \mathrm{db}$ and we also imposed the robustness requirement 
Table 2. Optimal Tuning Parameters

\begin{tabular}{ll}
\hline Parameter & Value \\
\hline$K_{p}$ & 50.5197 \\
$K_{i}$ & 151.0551 \\
$K_{d}$ & 0.0756 \\
$\lambda$ & 0.9170 \\
$\mu$ & 0.7590 \\
\hline
\end{tabular}

$\zeta=1 \mathrm{deg} \cdot \mathrm{rad}^{-1} \cdot$ day. The optimal tuning parameters of the fractional controller are given in Table 2. The optimal value of $J_{\text {itae }}$ following the excitation of the system with a step pulse of amplitude $x^{\mathrm{sp}}=+0.1$ was found to be 3.9713 . $10^{-4} \mathrm{ng}$. Using the Bode diagram of $G_{\mathrm{ol}}$ shown in Fig 4

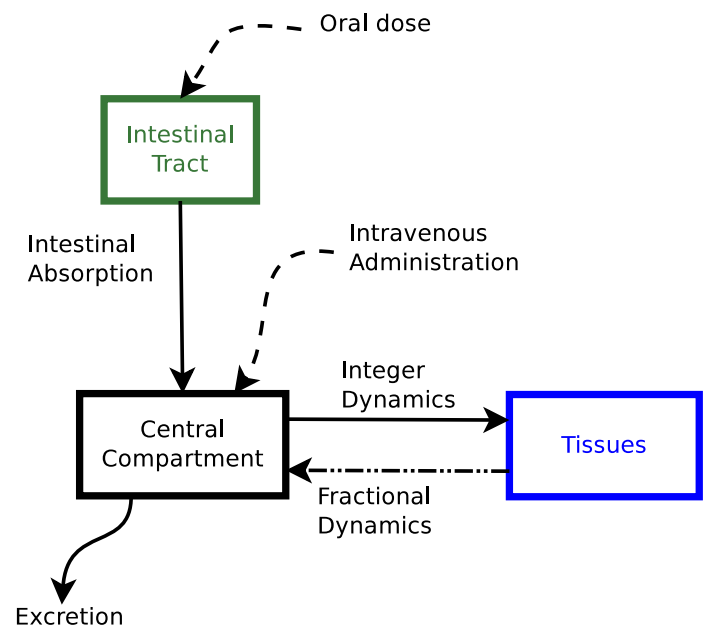

Fig. 1. Topology of the pharmacokinetic system used for the derivation of the pharmacokinetic model (20).

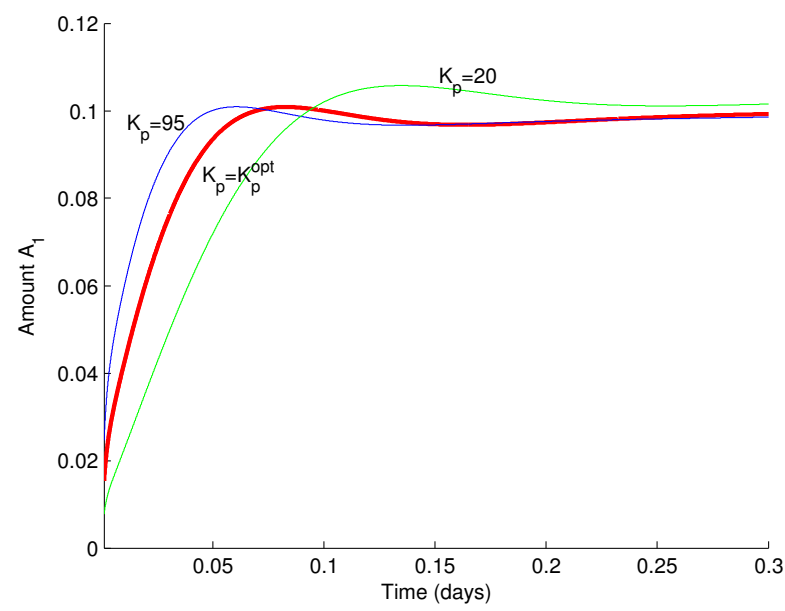

Fig. 2. Step-response of the closed-loop system for a change of the set-point by +0.1 for various values of $K_{p}$. The thick red line corresponds to the optimal value $K_{p}^{\star}=50.52$. The amount of Amiodarone is in ng.

we calculated the phase and gain margins of the closed loop as well as the crossover frequency. A gain margin as high as $43.9 \mathrm{db}$ should be considered excellent and implies that the closed loop will remain stable even in presence of delay element as it is well away from instability. We may

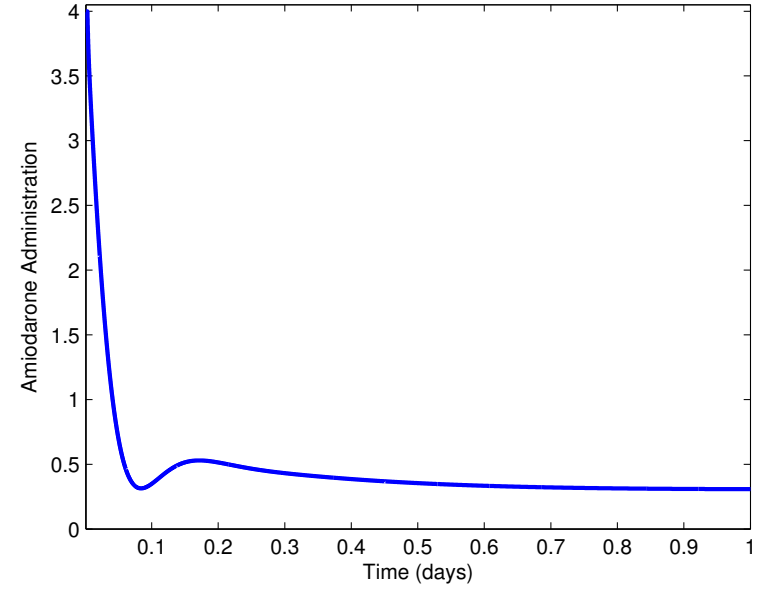

Fig. 3. The control action produced by the optimally tuned fractional controller. The administration rate is in ng/day.

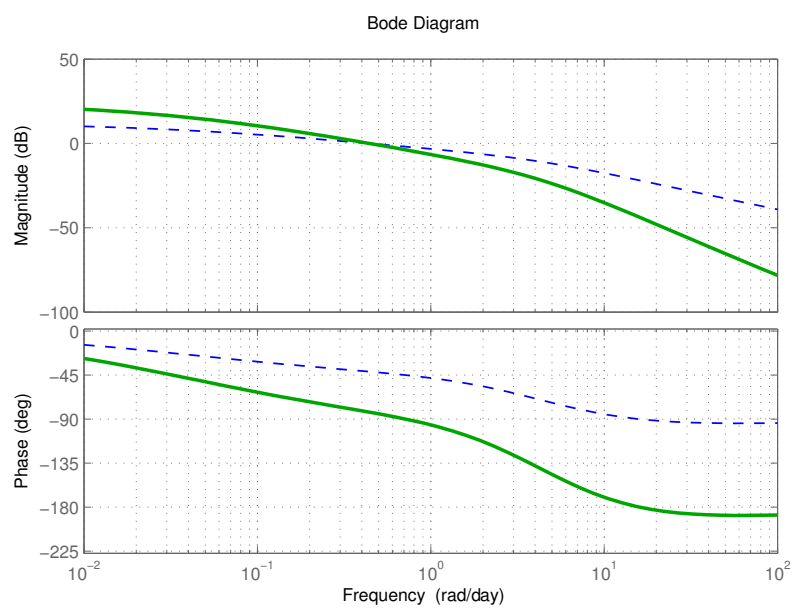

Fig. 4. Bode diagram: The blue dashed line corresponds to the pharmacokinetic system and the thick green solid line to the open-loop system in presence of the optimally tuned $\mathrm{PI}^{\lambda} \mathrm{D}^{\mu}$ controller. The phase margin is $98^{\circ}$ and the gain margin is $43.9 \mathrm{db}$. The crossover frequency is $16 \mathrm{rad} /$ day. It was found that $M_{z}=$ $0.5 \mathrm{deg} \cdot \mathrm{rad}^{-1} \cdot \mathrm{day}$

draw the same conclusion observing the high phase margin of $98^{\circ}$. The phase margin translates into the maximum delay that the closed-loop can take remaining stable; this is calculated by the formula:

$$
\tau_{d, \max }=\frac{M_{p}}{\omega_{1}}=3.37 \text { day }
$$

where $\omega_{1}=0.43 \mathrm{rad} /$ day is the frequency at which $\left|G_{\mathrm{ol}}\left(\imath \omega_{1}\right)\right|=0 \mathrm{db}$ and $M_{p}$ is the phase margin in rad.

As one can see in Fig. 5, the closed-loop transfer function exhibits very low gain $(<-60 \mathrm{db})$ at high frequencies $\left(>\omega_{h}\right)$, thus filtering out high-frequency noise signals in the closed loop and/or noise that comes with the set-point. Despite of the quite high values of the stability margins, the system responds satisfactorily fast. Due to the high gain margin, we can increase the controller's gain so as 


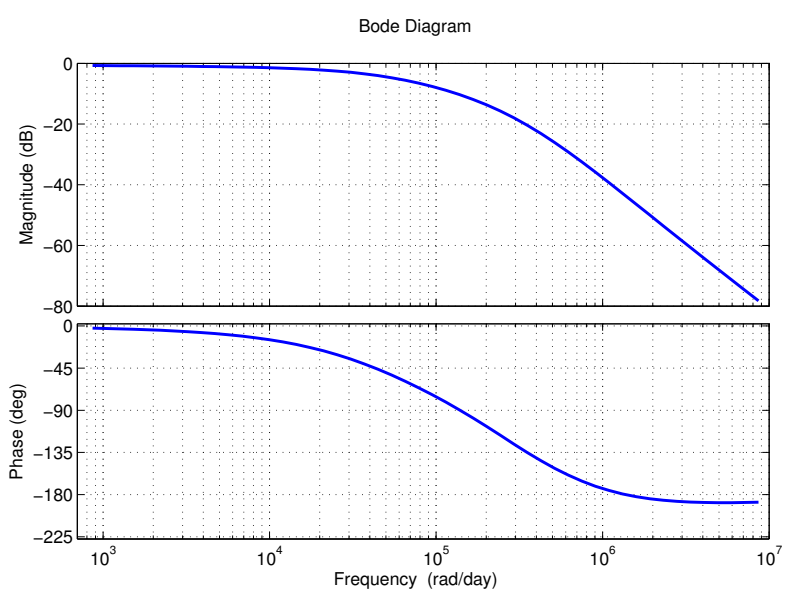

Fig. 5. Bode diagram of the closed-loop transfer function. Note that at high frequencies the gain is lower than $-60 \mathrm{db}$, thus, the controller attenuates the highfrequency noise in the closed-loop or possible noise that comes with $x^{\mathrm{sp}}$.

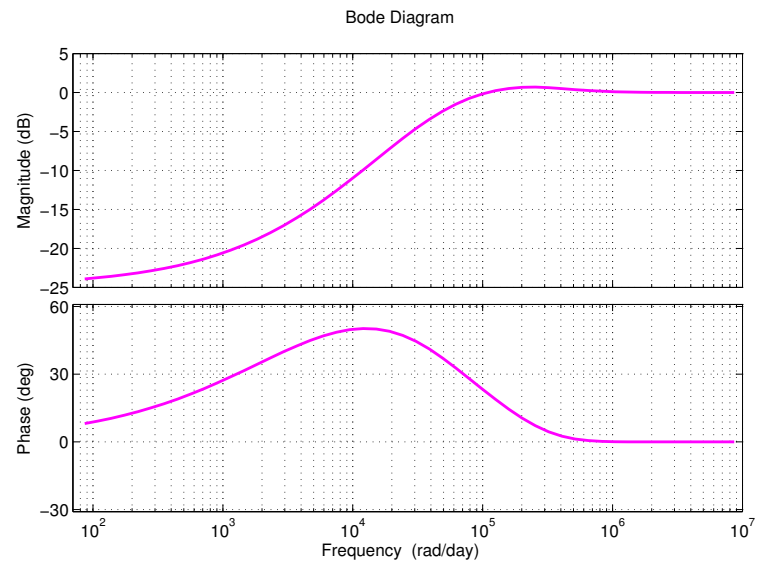

Fig. 6. Bode diagram of the sensitivity function of the system.

to obtain faster responses at the cost of a higher ITAE value. The sensitivity function of the system exhibits low gain (lower than -20db) at low frequencies as one can see in Fig. 6 which, in turn, implies that the system can attenuate output disturbacens.

All time-domain simulations were performed using an Oustaloup filter in the frequency range $10^{-3} \sim 10^{3} \mathrm{rad} /$ day. For the frequency-domain calculations no approximation was necessary.

\section{CONCLUSION}

Fractional Systems theory enables us to model nonlinear processes with (infinite) memory such as anomalous diffusion and deep tissue trapping and presents great perspectives for pharmacokinetic modelling. Such systems can be used for the formulation of control problems where the goal is to maintain a constant drug concentration in a tissue of organ of the body.
In this paper we designed a fractional-order $\mathrm{PI}^{\lambda} \mathrm{D}^{\mu}$-type controller for the stabilisation of the concentration of Amiodarone whose pharmacokinetic distribution in the organism is described by a fractional-order model and we presented closed-loop simulations. The designed controller not only has a high gain margin but also exhibits excellent behaviour with respect to noise rejection in the closed loops and attenuation of externally applied disturbances. The proposed control approach is a good candidate for the control of other drugs that follow such fractionalorder dynamics such as Mibefradil (Fuite et al. [2002]) or Diclofenac (Popović et al. [2010]).

\section{REFERENCES}

M. Aoun, R. Malti, F. Levron, and A. Oustaloup. Numerical simulations of fractional systems: An overview of existing methods and improvements. Nonlinear Dynamics, 38(1-4):117-131, 2004.

Karl Johan Åström. Control system design. Lecture Notes for ME 155A, 2002.

A. Dokoumetzidis and P. Macheras. IVIVC of controlled release formulations: Physiological-dynamical reasons for their failure. Journal of Controlled Release, 129(2): 76-78, 2008.

A. Dokoumetzidis and P. Macheras. Fractional kinetics in drug absorption and disposition processes. Journal of Pharmacokinetics and Pharmacodynamics, 36:165-178, 2009.

A. Dokoumetzidis, R. Magin, and P. Macheras. Fractional kinetics in multi-compartmental systems. Journal of Pharmacokinetics and Pharmacodynamics, 37:507-524, 2010a.

A. Dokoumetzidis, R. Magin, and P. Macheras. A commentary on fractionalization of multi-compartmental models. Journal of Pharmacokinetics and Pharmacodynamics, 37:203-207, 2010b.

P. Dua, F.J. Doyle, and E.N. Pistikopoulos. Model-based blood glucose control for type 1 diabetes via parametric programming. Biomedical Engineering, IEEE Transactions on, 53(8):1478-1491, aug. 2006. ISSN 0018-9294. doi: 10.1109/TBME.2006.878075.

J. Fuite, R. Marsh, and J. Tuszynski. Fractal pharmacokinetics of the drug mibefradil in the liver. Phys Rev E Stat Nonlin Soft Matter Phys, 66, August 2002.

R. Hilfer. Fractional diffusion based on riemann-liouville fractional derivatives. J. Phys. Chem. B, 104:3914-3917, 2000a.

R. Hilfer. Applications Of Fractional Calculus In Physics. World Scientific, 2000b. ISBN 978-981-02-3457-7.

V. Kühlkamp, C. Mewis, R. Suchalla, J. Mermi, V. Dörnberger, and L. Seipel. Effect of amiodarone and sotalol on the defibrillation threshold in comparison to patients without antiarrhythmic drug treatment. International Journal of Cardiology, 69(3):271-279, 1999.

J. Kytariolos, A. Dokoumetzidis, and P. Macheras. Power law IVIVC: An application of fractional kinetics for drug release and absorption. European Journal of Pharmaceutical Sciences, 41(2):299-304, 2010.

J. T. Machado, V. Kiryakova, and F. Mainardi. Recent history of fractional calculus. Communications in Nonlinear Science and Numerical Simulation, 16(3):11401153, 2011. 
R. Magin. Fractional Calculus in Bioengineering. Begell House Publ., 2006. ISBN 978-1-56700-215-7.

Farshad Merrikh-Bayat. Rules for selecting the parameters of oustaloup recursive approximation for the simulation of linear feedback systems containing $P I^{\lambda} D^{\mu}$ controller. Communications in Nonlinear Science and Numerical Simulation, 17(4):1852-1861, 2012. ISSN 1007-5704. doi: $10.1016 /$ j.cnsns.2011.08.042.

Concepción A. Monje, YangQuan Chen, Blas M. Vinagre, Dingyü Xue, and Vicente Feliu. Fractional-order proportional integral controller tuning for first-order plus delay time plants. In Fractional-order Systems and Controls, Advances in Industrial Control, pages 87-106. Springer London, 2010. ISBN 978-1-84996-334-3. doi: 10.1007/978-1-84996-335-0_5.

A. Oustaloup, F. Levron, B. Mathieu, and F.M. Nanot. Frequency-band complex noninteger differentiator: characterization and synthesis. Circuits and Systems I: Fundamental Theory and Applications, IEEE Transactions on, 47(1):25-39, January 2000. ISSN 1057-7122. doi: $10.1109 / 81.817385$.

L. M. Pereira. Fractal pharmacokinetics. Comput. Math. Methods, 11:161-184, 2010.

I. Podlubny. Fractional-order systems and $P I^{\lambda} D^{\mu}$ controllers. IEEE Transactions on Automatic Control, 44(1):208-214, January 1999.

J. K. Popović, M. T. Atanacković, A. S. Pilipović, M. R. Rapaić, S. Pilipović, and T. M. Atanacković. A new approach to the compartmental analysis in pharmacokinetics: fractional time evolution of diclofenac. Journal of Pharmacokinetics and Pharmacodynamics, 37:119-134, 2010.

J. K. Popović, M. Poša, K. J. Popović, D. J. Popović, N. Milošević, and V. Tepavčević. Individualization of a pharmacokinetic model by fractional and nonlinear fit improvement. European Journal of Drug Metabolism and Pharmacokinetics, pages 1-8, 2012.

H. Sarimveis, P. Sopasakis, A. Afantitis, and G. Melagraki. A model predictive control approach for optimal drug administration. Chemical Engineering Transactions, 17, 2009.

P. Sopasakis and H. Sarimveis. An integer programming approach for optimal drug dose computation. Computer Methods and Programs in Biomedicine, 108(3):1022-1035, 2012. ISSN 0169-2607. doi: 10.1016/j.cmpb.2012.06.008.

Duarte Valério and José Sá da Costa. Tuning of fractional PID controllers with Ziegler-Nichols-type rules. Signal Processing, 86(1), 2006.

D. Verotta. Fractional dynamics pharmacokineticspharmacodynamic models. Journal of Pharmacokinetics and Pharmacodynamics, 37:257-276, 2010a.

D. Verotta. Fractional compartmental models and multiterm Mittag-Leffler response functions. Journal of Pharmacokinetics and Pharmacodynamics, 37:209-215, 2010b. 